Figure 2. Comparison of different sources of variance in Earth's recent surface (left) and lower stratospheric (right) temperatures, deduced from multivariate linear regression. The volcanic aerosols and ENSO indices are lagged by 6 months, and solar irradiance by one month, relative to the observed surface temperatures, but in the lower stratosphere all lags are zero.
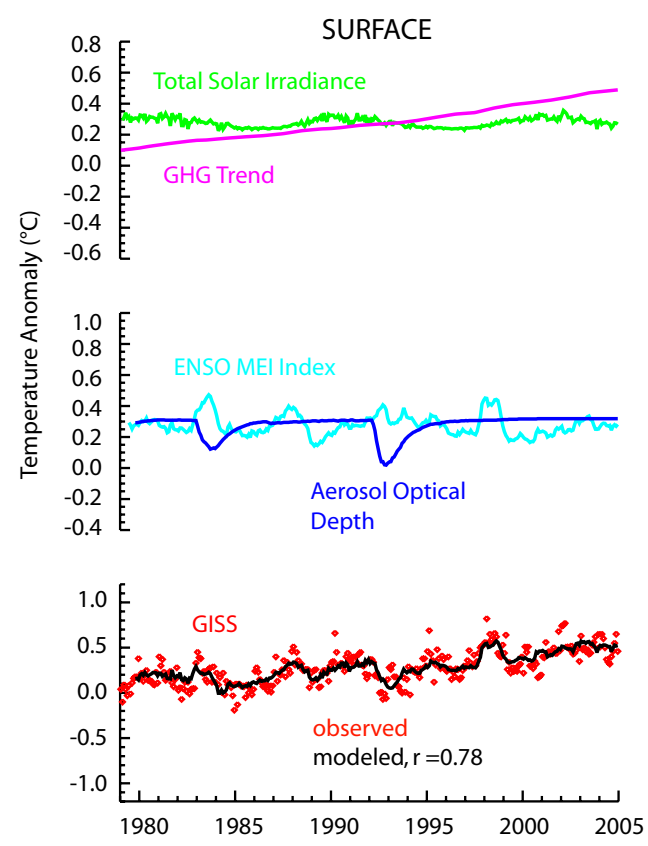

LOWER STRATOSPHERE
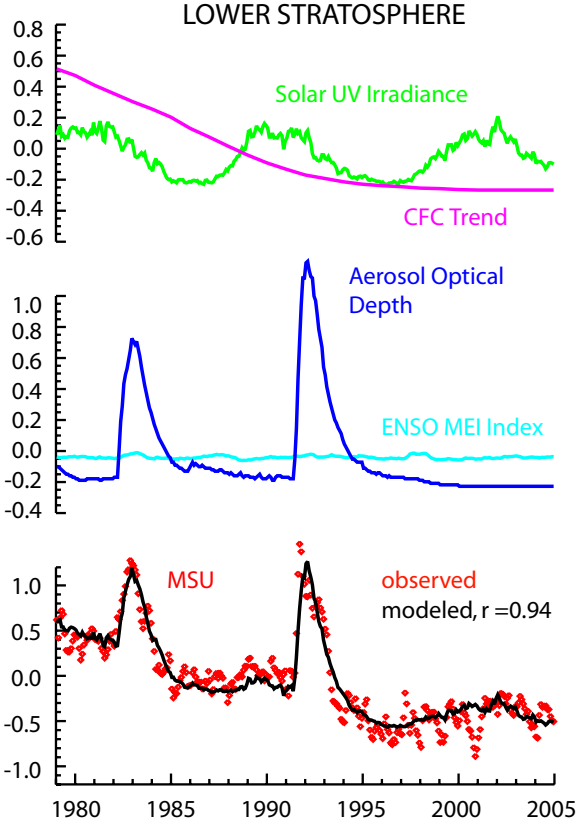

Lean, J., Rottman, G., Harder, J., and Kopp, G.: 2005, SORCE contributions to new understanding of global change and solar variability, Solar Phys., in press.

Rind, D.: 2002, The Sun's role in climate variations, Science
Wang, Y-. M., Lean, J. L., and Sheeley, Jr., N. R.: 2005, Modeling the Sun's magnetic field and irradiance since 1713, Astrophys. J. 625, 522-538.

296, 673-677.

\title{
Aerosol effects on clouds and climate
}

\section{Ulrike Lohmann}

\section{Institute for Atmospheric and Climate Science, ETH Zürich, Zurich, Switzerland; ulrike.Iohmann@env.ethz.ch}

The burning of fossil fuels and biofuels due to human activities has greatly increased the amount of particulate matter in the atmosphere. The major aerosol components are mineral dust, sea salt, sulfates, nitrates, black carbon (also termed soot) and particulate organic matter (POM). The natural aerosol species, mineral dust and sea salt, dominate the mass concentration in the atmosphere. On average, they contribute $39 \mathrm{mg} / \mathrm{m}^{2}$ and $13 \mathrm{mg} / \mathrm{m}^{2}$, respectively, whereas the anthropogenic components, sulfate, POM and black carbon only contribute 3.9, 3.3 and $0.4 \mathrm{mg} / \mathrm{m}^{2}$, respectively, to the annual global average as deduced from 20 different global models (Kinne et al., 2005). So far, nitrate is not included in most models because of its semi-volatile nature.

Optically, mineral dust and sea salt are less important because of their larger size. Thus, they each contribute only as much to the aerosol optical depth as sulfate does $(25 \%)$. Black carbon, which contributes only $3 \%$ to the optical depth, is the main aerosol type that absorbs solar radiation and can lead to a warming of the surrounding air. This warming can prevent cloud formation because the atmosphere becomes more stable, or even lead to an evaporation of cloud droplets. This semi-direct effect thus counteracts some of the negative aerosol forcings from scattering aerosols, such as sea salt and sulfate, at the top of the atmosphere (e.g., Lohmann and Feichter, 2005).

At the Earth's surface, however, both scattering and absorbing aerosols work in the same direction to reduce the amount of solar radiation reaching the surface. Since pre-industrial times, increasing emissions of aerosols as a result of human activity have caused a reduction of solar radiation at the surface ("solar dimming") by increasing aerosol and cloud optical depth. Such a reduction of $1.3 \%$ per decade over the land surfaces from 1961 to 1990 has been observed in many regions worldwide (e.g., Wild et al., 2004). Recent surface observations, however, show that the long term decline in solar radiation at land surfaces turned into an increase in surface solar radiation during the 1980s (Wild et al., 2005), in agreement with recent emission trends in the "old" industrial regions in the northern hemisphere (Krüger and Graßl, 2002), as well as with long-term black carbon trends in the Canadian Arctic (Sharma et al., 2004), and sulfate deposition declines over Europe and North America since 1978 (E. Holland, pers. comm.).

Aerosols also act as centres for cloud droplets and ice crystals, thereby changing cloud properties. If more aerosols compete for the uptake of water vapour, the resulting cloud droplets do not grow as large. More smaller cloud droplets have a larger surface area than fewer larger cloud droplets for the same amount of cloud water. Thus, a polluted cloud reflects more solar radiation back to space, resulting in a negative radiative forcing at TOA (cloud albedo effect). In addition, these more numerous but smaller cloud droplets collide less efficiently with each other, which reduces the precipitation efficiency of polluted clouds and prolongs their lifetime. It also implies more scattering of solar radiation back into space, thus reinforcing the cloud albedo effect. Whether the cloud lifetime or the cloud albedo effect is more important is still an open question. Whereas some models predict that the cloud albedo effect is four times as 
important as the cloud lifetime effect, other models predict that the cloud lifetime effect dominates over the cloud albedo effect (Fig. 1).

The global mean magnitude of the cloud albedo effect since pre-industrial times is estimated between -0.5 and $-1.9 \mathrm{~W} / \mathrm{m}^{2}$ from different climate models and the cloud lifetime effect to be between -0.3 and $-1.4 \mathrm{~W} / \mathrm{m}^{2}$ (Lohmann and Feichter, 2005). The semi-direct effect, which could in principle counteract part of this negative forcing at TOA, is predicted to be only between -0.5 and $+0.1 \mathrm{~W} / \mathrm{m}^{2}$, where the negative values result from black carbon being located above the cloud. If the individual indirect effects values are summed up, the indirect effect could amount to almost -3 $\mathrm{W} / \mathrm{m}^{2}$. This exceeds estimates from simple inverse models that start from the observed land temperature rise and increased ocean heat uptake in the 20th century, which bracket the overall indirect aerosol effect to be between 0 and $-2 \mathrm{~W} / \mathrm{m}^{2}$ (Anderson et al., 2003). Thus, either climate model predictions of the cloud albedo and / or cloud lifetime effect are too large, or a counteracting effect is missing.

A proposed counteracting effect could include the ice phase (glaciation effect). Here increases in ice nuclei in the present-day climate result in more frequent freezing of supercooled clouds. As the precipitation formation in ice clouds is faster than in water clouds, this would increase the overall amount of precipitation. A climate model prediction including this effect resulted in reduced cloud cover at mid and high latitudes of the northern hemisphere and more solar radiation absorbed within the Earth-atmosphere system, thus partly offsetting the indirect effects on warm clouds (Lohmann, 2002). Other possibilities refer to aerosol effects on convective clouds (Rosenfeld and Woodley, 2000) that have not been considered in climate models so far.

\section{References}

Anderson, T.L., R.J. Charlson, S.E. Schwartz, R. Knutti, O. Boucher, H. Rodhe, and J. Heintzenberg, 2003: Climate forcing by Aerosols - a hazy picture. Science, 300, 1103-1104.

Kinne S., et al., 2005: An AeroCom initial assessment - optical properties in aerosol component modules of global models, Atmos. Chem. Phys. Disc., submitted.

Krüger, O. and H. Graßl, 2002: The indirect aerosol effect over Europe. Geophys. Res. Lett., 29, doi: 10.1029/2001GL014081.

Lohmann, U., 2002: A glaciation indirect aerosol effect caused by soot aerosols. Geophys. Res. Lett., 29, doi: 10.1029/2001GL014357.

Lohmann, U. and J. Feichter, 2005: Global indirect aerosol effects: A review. Atmos. Chem. Phys., 5, 715-737.

Rosenfeld, D. and W. L. Woodley, 2000: Deep convective clouds with sustained supercooled liquid water down to $-37.5^{\circ} \mathrm{C}$. Nature, $405,440-442$.

Sharma, S., D. Lavoué, H. Cachier, L.A. Barrie, and S.L. Gong, 2004: Long-term trends of the black carbon concentrations in the Canadian Arctic. J. Geophys. Res., 109, doi: 10.1029/2003JD004331.

Wild, M., A. Ohmura, H. Gilgen, and D. Rosenfeld, 2004: On the consistency of trends in radiation and temperature records and implications for the
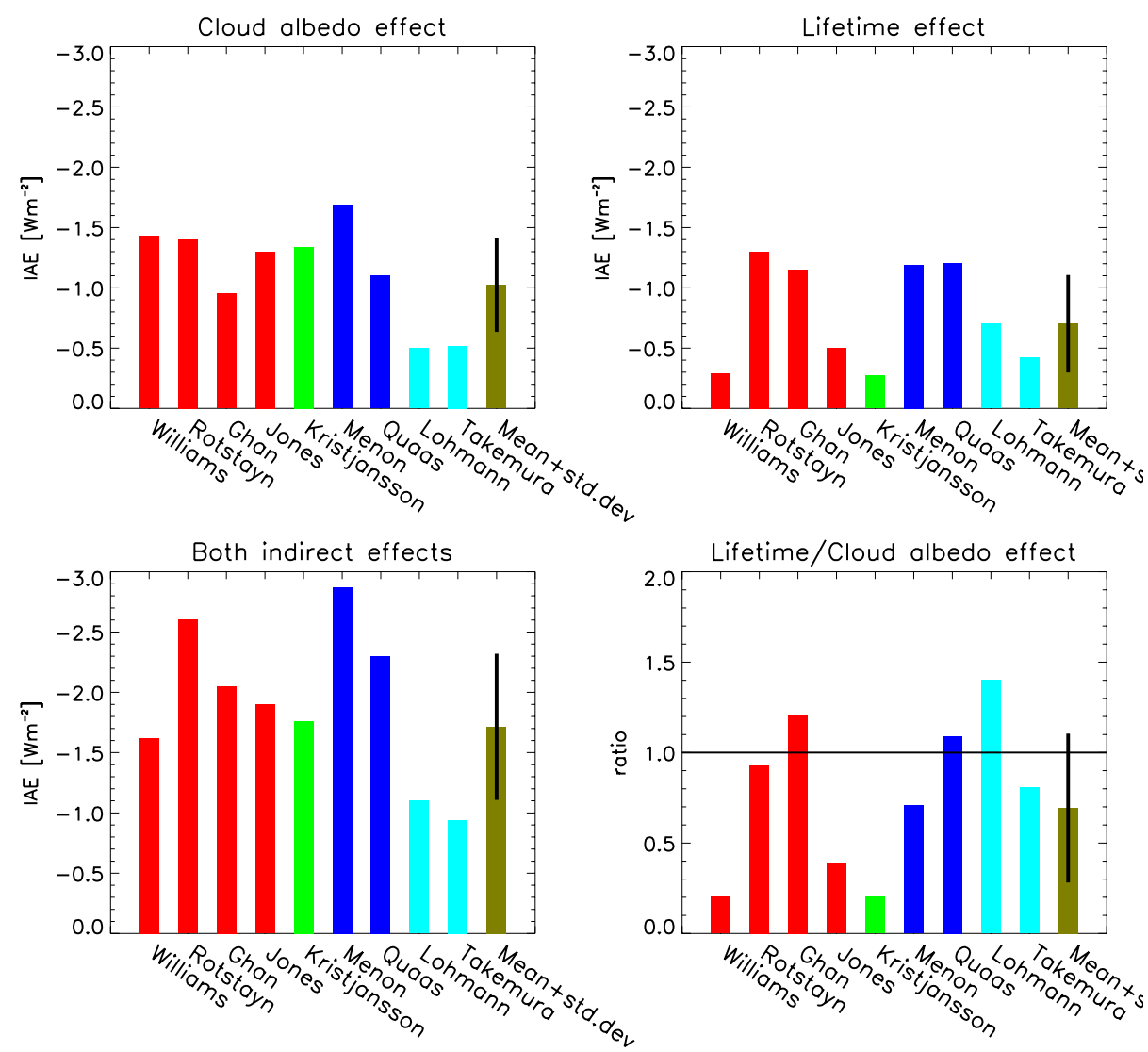

Figure 1: Global mean cloud albedo effect, lifetime effect, both effect and the ratio lifetime effect/cloud albedo effect for different models considering only anthropogenic sulfate aerosols (red bars), anthropogenic sulfate and black carbon (green bars), anthropogenic sulfate and organic carbon (blue bars), anthropogenic sulfate, and black and organic carbon (turquoise bars) and the mean plus standard deviation from all simulations (olive bars), adapted from Lohmann and Feichter (2005). 
global hydrological cycle. Geophys. Res. Lett., 31, doi: 10.1029/2003GL019188.

Wild, M., H. Gilgen, A. Rösch, A. Ohmura, C. N. Long, E. G. Dutton, B. Forgan, A. Kallis, V. Russak, and
A. Tsvetkov, 2005: From dimming to brightening: Decadal Changes in Solar Radiation at Earth's Surface, Science, 308, 847-850.

\section{Mineral dust records from Greenland ice cores}

\section{Urs Ruth}

\section{Alfred-Wegener-Institut für Polar- und Meeresforschung, Bremerhaven, Germany; uruth@awi-bremerhaven.de}

Mineral dust plays an important role in climate research today. Through its radiative properties, mineral dust aerosol directly influences the radiative balance of the atmosphere. Furthermore, mineral dust is believed to be climatically active in a number of indirect ways. These include its ability to act as cloud-condensation nuclei with consequences for the amount and characteristics of cloud cover, as well as its potential to control primary bioproductivity in remote ocean and land areas that would otherwise be nutrient limited (fertilisation). Although not all of these processes are yet well understood the great dynamics of the mineral dust cycle in the past allows for an important feedback loop for climatic changes. On this question it is not yet certain whether the great concentration increase during glacial times, as observed in polar ice cores, is restricted to the high latitudes or whether it is representative for larger parts of the atmosphere.

Terrestrial, marine and ice-core archives of windblown mineral dust can be used to infer past climate conditions relevant to the dust cycle. For this purpose, it is very fortunate that - at least for Greenlandic ice cores-the source regions of mineral dust have been very well localised: The East Asian deserts in Western China and Inner Mongolia have been identified through isotopic and mineralogical studies as predominant sources for recent times as well as for the last glacial period (Fig. 1) (Svensson et al., 2000, Bory et al., 2003). Therefore, dust from ice cores is distinct from most other proxy parameters for which the sources are neither well known nor localised in space. Accordingly, an idealised concept for interpreting Greenlandic ice core dust records consists of (i) mobilisation of dust in the source and uplift into the free troposphere, (ii) long-range transport with the westerly jets over a source-free area, (iii) loss processes en route due to gravitational settling and wash-out, and (iv) deposition onto the ice sheet. The total mineral dust load is divided into a soluble fraction, which is best approximated by $\mathrm{Ca}^{2+}$ ion concentrations, and an insoluble fraction, which for practical reasons is most commonly inferred from the volume of water-insoluble particles in molten ice-core samples. Usually, both fractions vary alike and point to only small though significant compositional variations. Apart from the concentration, the size distribution of insoluble dust particles can also be measured, which provides unique information on changes of past atmospheric long-range transport.

Continuous time series of insoluble dust particle concentration and size distribution from the North-GRIP ice core are shown in Figure 2 (Ruth et al., 2003). The concentration exhibits a strong interglacial-glacial increase by a factor of $\sim 100$ and an interstadial-stadial increase by a factor of typically $\sim 10$. The changes are very rapid and therefore point to atmospheric processes as their main drivers. The two most likely mechanisms to produce high ice core dust levels are (a) increased mobilisation in the source area due to higher wind speeds or more frequent dust storms, and (b) increased long-range transport efficiency due to shorter transit times or less wash-out en route. It is a matter of debate which one of these mechanisms is the more important, with considerable paleoclimatic implications: If the observed increase was primarily due to more efficient long-range transport then the large increase would be limited to the remote regions; if however the increased dust flux was primarily due to increased source strength, then the increase would scale proportionally along the whole atmospheric transport pathways.

A first indication can be deduced from the ice core dust record itself: The size distribution-here given as the lognormal mode (i.e. maximum) of the volume distribution-shows larger particles during colder periods. This indicates shorter transport durations during colder periods because larger particles undergo stronger gravitational settling than small ones and are thus less depleted during times of short transport durations. Given the stationary location of the sources, a shorter transport duration implies faster transporting winds unless the pathway has changed. Quantitative estimates based on a simple 1-D transport model suggest a reduction of transport time by $\sim 25 \%$ for LGM compared to Holocene and by $\sim 10 \%$ for stadials compared to interstadials. Assuming exponential decrease of concentration during transport and a decrease of the hydrological cycle during LGM by a factor of two, this implies concentration increases by a factor of $\sim 4$ and $\sim 2$ for Holocene/LGM and interstadial/stadial, respectively (Ruth et al., 2003). Thus, only a small fraction of the observed concentration increase can be explained by enhanced transport efficiency during cold times.

Another indication can be obtained from Chinese loess archives, which are close to the source areas for Greenland ice core dust and are influenced by the same monsoonal circulation regimes. The mean diameter of loess grains is indicative of the strength of the transporting winds, which are expected to correlate with the strength of dust storms that cause the mobilisation of Greenland ice core dust. Therefore, the grain size record of the Luochuan loess section (Xiao et al., 1999) is compared to the NorthGRIP dust concentration in Figure 2. Despite its inferior time resolution, it shows remarkable similarities with the North-GRIP particle concentration. It is difficult to quantify how a change in loess granulometry should relate to the source strength for Greenland ice core dust. However, the data together with our knowledge about present-day climatology suggests a causal link between regional loess transport within China and dust mobilisation for intercontinental export of dust during the last glacial period. This notion is supported by the observed increase 